\title{
Decision Intelligence in Street Lighting Management
}

\author{
Diogo Nunes ${ }^{1}$, Daniel Teixeira ${ }^{1}$ Davide Carneiro ${ }^{1,2}$, Cristóvão Sousa ${ }^{1,3}$, and \\ Paulo Novais ${ }^{2}$ \\ 1 CIICESI/ESTG, Polytechnic Institute of Porto, Portugal \\ $\{8140365,8140360$, dcarneiro, cds\}@estg.ipp.pt \\ 2 INESC TEC, Porto, Portugal \\ 3 Algoritmi Center/Department of Informatics, University of Minho, Portugal \\ pjon@di.uminho.pt
}

\begin{abstract}
The European Union has been making efforts to increase energy efficiency within its member states, in line with most of the industrialized countries. In these efforts, the energy consumed by public lighting networks is a key target as it represents approximately $50 \%$ of the electricity consumption of European cities. In this paper we propose an approach for the autonomous management of public lighting networks in which each luminary is managed individually and that takes into account both their individual characteristics as well as ambient data. The approach is compared against a traditional management scheme, leading to a reduction in energy consumption of $28 \%$.
\end{abstract}

Keywords: smart cities, intelligent system, energy management

\section{Introduction}

Cities currently face several challenges such as environmental stressors, overpopulation, and other related issues (e.g. traffic problems, air pollution). In the search for technological solutions for these problems, the so-called Smart Cities emerged [2].

At the most basic level, a Smart City implies the use of Information and Communication Technologies (ICT) to connect its different services and/or resources, allowing data to be collected, analyzed and acted upon in real time [3]. However, the simple interconnection of these elements is not enough to make a city "Smart". Thus, a Smart City also implies the use of the collected data with the goal to improve the citizens' quality of life [4], the efficiency of the city management [8], the management of resources, and the sustainability of the city and its growth [6]. Hence, and as put argued by Mumford [10], the challenges posed by smart city projects are, typically, socio-technical in nature.

One particularly important element of life in a city is public lighting: it contributes to citizen's comfort, safety and perceived security [9]. At the same time, it has also a significant economic impact in the municipalities' expenditures 
with energy. In Portugal, the latest data show that public lighting amounts to $3 \%$ of the country's expenses with energy[12].

Current technological developments allow the development of solutions to minimize energy consumption. In this paper we propose such a solution, validated in a network of more than 300 luminaries, also considering guidelines and legislation concerning the ideal levels of light in public spaces.

To this end, we explore two main aspects. First, each luminary is managed individually. Indeed, in previous work we have shown how different luminaries of the same model behave differently in terms of energy consumption or running temperature [7]. This can be explored to optimize each luminary individually, taking into consideration its characteristics. Second, we use data about ambient luminosity in order to dim intensity in real-time for each individual luminary.

The proposed system was developed and validated in a test setting, and then extrapolated to 4 months of data collected from a public lighting network with 305 luminaries. When compared to the scheme that is currently used by the municipality to manage this lighting network, the use of the proposed system would lead to a decrease in energy consumption of $28 \%$.

\section{Energy Consumption Efficiency in the Context of Street Lighting}

Energy issues continue on the agenda, in particular in what concerns to consumption efficiency aspects. In fact, we are witnessing a transitional period, wherein existing renewable and clean energy sources are not enough or are not sufficiently mature to answer current global demands. Meanwhile, new solutions focused on redesigning energy consumption patterns emerge, in order to overcome sustainability unbalance. That is what Smart cities stand for, that is, they are on pursuit of new resource management through digital sustainability approaches. Within this context, cities' street lighting network has been shifting towards a new technology paradigm that allows it to benefit from significant energy savings. However, despite of the economic return, energy consumption efficiency is not guaranteed. Energy efficiency in the context of street lighting is a broader concept and socio-technical in nature. Measuring energy consumption efficiency in a decision support perspective, implies to understand energy efficiency at its basis and in the socio-economic perspective.

\subsection{Understanding energy efficiency panorama}

The European Union has been making efforts to increase energy efficiency within its member states. The 2030 climate and energy framework, revised in 2018 for the period from 2021 to 2030, aims the reduction of $32.5 \%$ in energy consumption replacing with $32 \%$ of the energy coming from renewable sources keeping the value of $40 \%$ in greenhouse gas emissions. Portugal has also defined its own energy strategy in accordance do the European Union commitment and developed 
the PNAEE (National Action Plan for Energy Efficiency), containing measures and guidelines to be followed in Portugal.

In the energy efficiency panorama, "lighting represents approximately 50\% of the electricity consumption of European cities" [13], endowing street lighting a crucial role on cities energy efficiency road map, whose guidelines were expressed in a set o documents/standards for road lighting (EN 13201:2015) [1].

\subsection{Energy efficiency semantic outlook}

The generic definition of energy efficiency is related to the use of the lowest possible energy to produce the same amount of services or useful output [11]. The idea is to minimize energy consumption without harming the service quality and user comfort. Following this, street lighting management optimisation is the core engine through which is possible to achieve energy consumption efficiency. Hence, the better energy efficiency is understood, the better the optimisation approach might be.

In this context, energy efficiency must be approached as an holistic concept [1] including several factors or parameters that might be classified as internal and external factors, respectively. Internal factors are related to current (I), tension (v), electric power (w), color and temperature. Additionally, external factors include ambient data [5] and the location of street lighting installation [1], as well as life quality parameters [4].

Figure 1 depicts a conceptual ontology for street lighting energy consumption, representing a common understanding upon the domain. It is grounded on the rationale that street LED luminaries have a consumption behaviour that may be classified as typical, atypical and ideal. This classification is obtained according to the luminary energy consumption pattern defined according to several factors: i) Savings, given by the lamp efficacy ratio; ii) internal factors such as dimming, temperature and power; iii) external factors (moonlight, weather, traffic, dust), and; iv) light quality factor, which depends on the location of the street light installation and the underlying illuminance pattern. The location is classified in EN 13201 according to the street utility and user needs, which by its turn has an associated illuminance pattern.

This conceptualisation effort followed and iterative and incremental process, supported by literature reviews, with sporadic interactions with domain experts. The conceptual ontology is to be further developed towards its formalisation. Meanwhile it contributes for: i) data interpretation; ii) classification of the consumption pattern within an holistic perspective. The formulation of useful decisions, typically composed of a chain of actions, must be aligned to the intended outcomes. Actions and outcomes relations are better understood when there is a shared vision of the domain. In this sense, the conceptualisation ease the classification of the "system status", promoting decisions utility. 


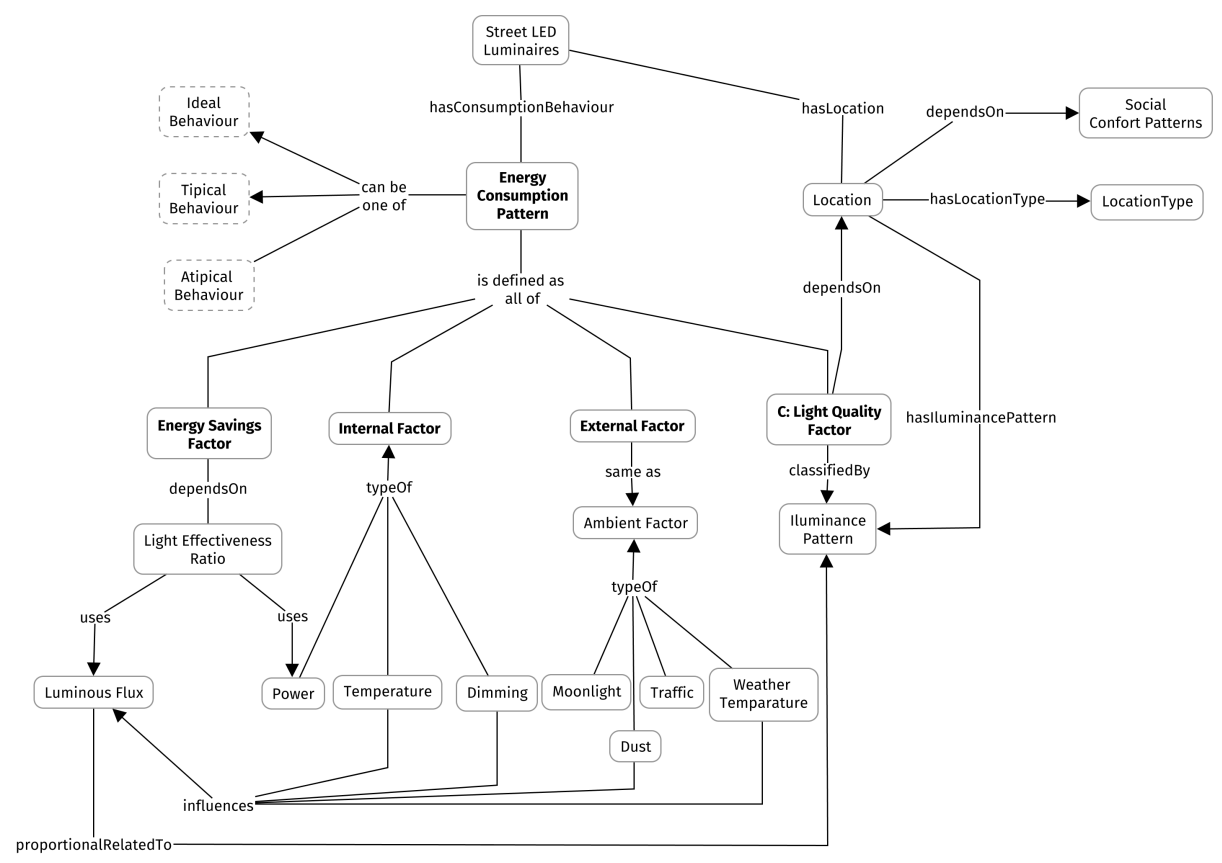

Fig. 1. Conceptual Ontology for street Lighting consumption

\section{Case Study}

\subsection{Data set Characterisation}

As mentioned earlier, providing smart decisions on how to minimize energy consumption requires contextualized data according to environmental factors (e.g., weather data, moonlight, traffic), normative factors and street lighting installation blueprint. The main source of data used in this work is a public lighting network in a production setting in a Portuguese municipality with 305 luminaries.

According to EN13201 normative, the set of luminaries were installed in a P2 zone, characterised as a pedestrian zone defined by horizontal illuminance patterns. The luminaries are distributed unilaterally in a 4 meter wide street plus 1 meter of sidewalk. To this type of location, the average lux should be 15 , with a minimum of 3 . In this scenario it is assumed that the luminaries are at 4 meters high, with 14,5 meters distance in between.

However, the data collected from this network was devoid from any kind of attributes than those related to internal factors. Thus, there was the need to enrich the available data-set with other relevant attributes, having into account the aforementioned domain model 1.

Thus, a second source of data was considered: a group of two luminaries in a laboratory setting and additional sensors. This setting, asides from freedom 
concerning the management of the luminaries, allowed us to deploy additional sensors to enrich the data collection process.

Both data sources and the data collected are described in Sections 3.2 and 3.3. In both settings the AQRUILED's ARQUICITY R1 luminary is used. This luminary allows for different data to be collected from its functioning in real time.

\subsection{Production Setting}

The dataset collected from the production setting contains 3.855.818 instances of data. Each instance describes 5 minutes of operation of a specific luminary and includes, among others, data about instant voltage, luminary temperature, instant power, accumulated energy (Wh), uptime or dimming. These data were collected over a period of four months, between the September 5th 2017 and January 3rd 2018.

The data from the luminaries was merged with weather data, also collected at 5-minute intervals from a local weather station. These data include air temperature $\left({ }^{\circ} \mathrm{C}\right)$, dew temperature $\left({ }^{\circ} \mathrm{C}\right)$, humidity $(\%)$, wind speed $(\mathrm{m} / \mathrm{s})$, wind direction (degrees), wind gust $(\mathrm{m} / \mathrm{s})$, pressure (mbar), solar irradiance $(\mathrm{W} / \mathrm{m} 2)$

and rain $(\mathrm{mm} / \mathrm{h})$. This allows to study the influence of external factors such as temperature on energy efficiency.

However, one of the limitations of this dataset is that it is a very homogeneous one. This derives from the fact that this is a production setting and must be managed according to the municipality's policies. For instance, $90 \%$ of the data was collected from luminaries set at a dimming between $80 \%$ and $90 \%$. This prevents us to explore the whole search space.

We hypothesize that the ideal value of dimming is not static, i.e., it varies according to the conditions of the environment, and that it may be outside of the $80 \%-90 \%$ interval. For that reason, data was also collected in the laboratory setting as described in the following section.

\subsection{Laboratory Setting}

Given the limitations of the data collected in the production setting described in the previous section, data was also collected in the laboratory setting. In this setting, data was also collected at 5-minute intervals from two luminaries in the test setting, between July 31st and October 4th, 2018.

These data include, like in the production setting, data from the luminaries and weather data. However, additional sensors were also used to collect data regarding luminosity at two different points (Figure 2): one at the pedestrian level and the other above the luminaries.

These data were then combined, which allows to study the influence of each of two key variables in the level of luminosity experienced by the pedestrian: dimming and ambient luminosity.

Moreover, the luminaries were programmed to change dimming at every 20 minutes, continuously changing between $50 \%$ and $100 \%$ with steps of $10 \%$. This 


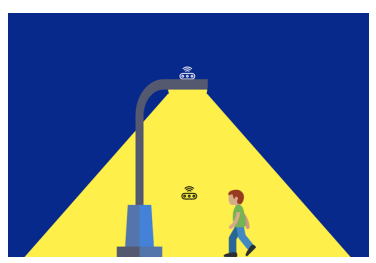

Fig. 2. Placement of the luminosity sensors in the laboratory setting.

provides a much more complete dataset about the luminaries' behavior in different schemes, widening the available search space.

Figure 3 shows how the luminosity measured under the luminary varies according to the dimming, without ruling out the effects of ambient light.

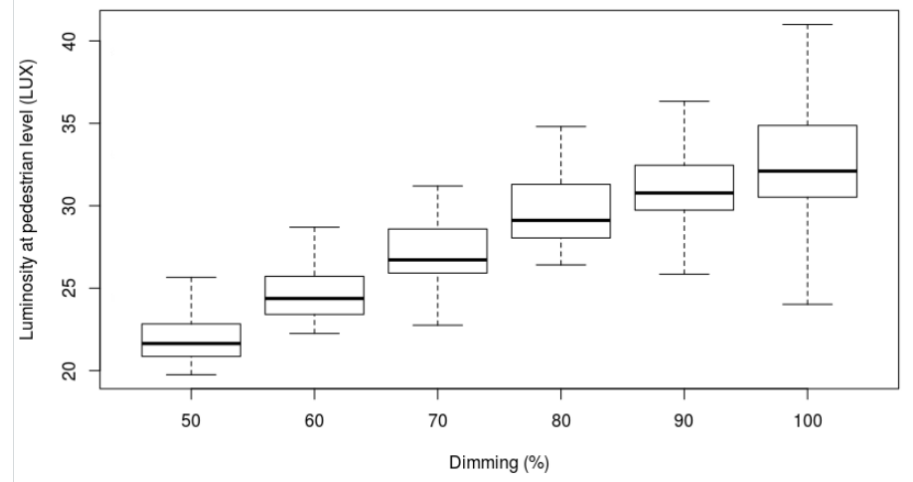

Fig. 3. Distribution of luminosity measured at pedestrian level in different dimmings, without ruling out the effect of ambient light.

\section{Implementation and Results}

As described in the Introduction section, the main goal of this work is to devise an approach for an individualized management of each luminary in a network, that takes into consideration the individual characteristics of each luminary as well as the intensity of ambient light in each region, in order to minimize energy consumption while maintaining a comfortable and safe level of lighting. To this end, the minimum level of luminosity was defined as 20 Lux, in accordance with the European Norm 13201, which defines the standards for road lighting.

To achieve the proposed goal, the following methodology was implemented. A Machine Learning model was trained with the dataset collected at the laboratory setting, whose aim is to predict the luminosity at pedestrian level, given the 
luminary characteristics (including dimming) and the ambient light. This model thus infers the influence of each variable (among which dimming and ambient light) on the level of luminosity experienced by a pedestrian. It then allows its use to estimate, for each luminary, the best configuration in order to minimize energy consumption while maintaining appropriate lighting levels.

Specifically, a Random Forest model was trained. This is one of the most popular ensemble learning algorithms due to its relative simplicity and its resilience to overfitting. In this algorithm, multiple Decision Trees are used as weak learners. These trees are purposely made simple during training, namely by limiting its depth (branching is stopped early) or by limiting the amount of data or the feature vector used in each tree. Each tree is thus trained on a different set of data, a process known as Bagging.

In this work, the resulting ensemble is composed of 50 trees, in which each tree was trained with $60 \%$ of the input variables, selected randomly. The ouput of the model is a numeric value that represents the predicted level of luminosity at pedestrian level, for a given scenario. The model exhibits an RMSE $=3.68$ $\left(r^{2}=0.53\right)$.

Figure 4 shows the observed value of luminosity at the pedestrian level for a randomly selected luminary/night, against the luminosity predicted by the model for the same data. The correlation between observed and predicted values is 0.89 . The variations in luminosity throughout the night are due to the ongoing changes in dimming in the operation of the luminaries of the laboratory setting, as described in Section 3.3, and to eventual natural changes in ambient light.

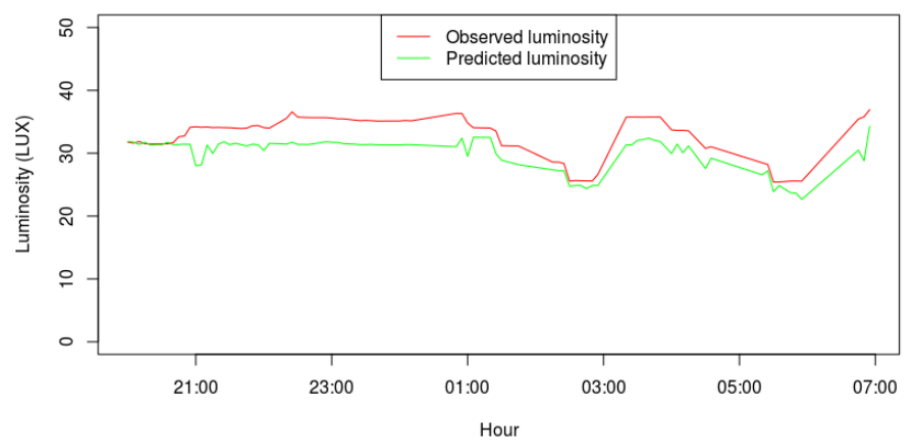

Fig. 4. Observed luminosity at pedestrian level for a randomly selected night, and luminosity predicted by the model $(\rho=0.89)$.

Figure 5 shows how the level of luminosity varies during one night selected randomly, and the prediction of the model for different levels of dimming. This Figure shows that, for this specific night, the optimum dimming would be around $60 \%$ as it provides the minimum desired level of luminosity according to the EN 13201 standard while minimizing energy consumption. 


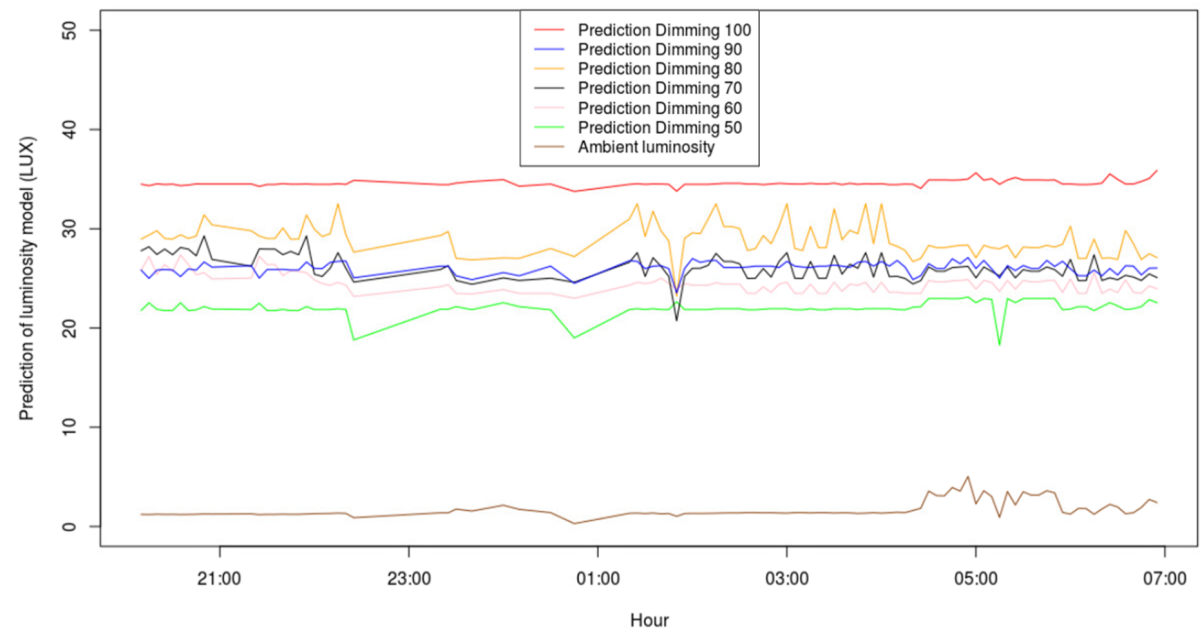

Fig. 5. Observed ambient luminosity (bottom line) and predicted luminosity at pedestrian level with different dimmings.

\subsection{Autonomous Luminary Management}

Once the model was trained, the next step was to devise a method to implement the autonomous management of the public lighting network to optimize energy consumption while maintaining light quality. The current scheme used by the municipality to manage the production setting is rather rigid: in weekdays the luminaries are set to $80 \%$ of dimming and in weekends they work at $100 \%$. This is hardly optimized for energy consumption. However, given that this is a production setting, we are not allowed to use this approach on site without prior validation.

To overcome this drawback, the following approach was implemented, with the goal to estimate the energy savings of implementing an optimized management scheme in the production setting. First, we must note that the luminaries in the production setting do not have ambient luminosity sensors. This prevents us to directly apply the model. In that sense, for each day of data in the production dataset, we randomly selected one day of ambient luminosity data from the test dataset, and combined them. The goal is, in the absense of luminosity data, to simulate it in a realistic manner.

With this, we are able to predict the luminosity at the pedestrian level. The next step was to devise a method for selecting the optimum dimming for each luminary. To this end, it is first necessary to predict power consumption from dimming. Since the power consumption in a luminary grows with dimming, a quadratic fit was calculated to model the relationship between the two variables (Figure 6). The resulting quadratic function can thus be used to predict the power consumption associated to a given dimming. 


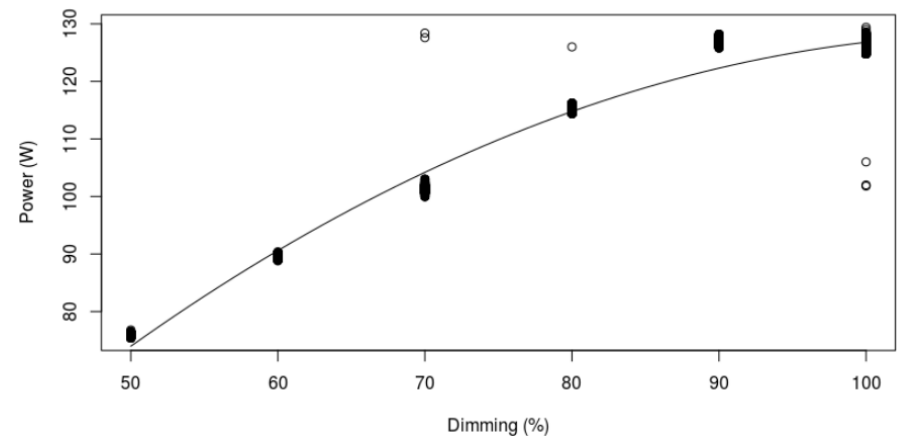

Fig. 6. Relationship between dimming and power consumption (RMSE = 1.95).

The following approach is implemented to select the optimum dimming for a specific luminary, at any given time. A binary search scheme is used for the interval 50\%-100\% dimming. Thus, it starts at a dimming of $75 \%$. For this dimming, and given the ambient light, both the power consumption and luminosity level at pedestrian level are estimated: the former using the quadratic regression, the latter using the trained model.

If the predicted luminosity is below the 20 Lux threshold established in the EN 13201 standard, the search continues to the right, i.e., at a dimming of $87.5 \%$. Otherwise, it continues to the left, at a dimming of $62.5 \%$. At each step, the estimations of power consumption and luminosity are updated. The process goes on until a value lower than $20 \mathrm{Lux}$ is reached and there is no possible search to the right. At this point, the previous value of dimming is selected.

This approach was used for every instance of data in the production dataset, in order to simulate the operation of an autonomous system carrying out a real-time management of each individual luminary, based on real-time data on ambient luminosity and luminary state. Results show a decrease in power consumption of around $28 \%$. The observed energy consumption over the 4 months of data was approximately $95.728 \mathrm{KW}$. In contrast, the estimated power consumption using the optimization scheme is of approximately $69.585 \mathrm{KW}$.

\section{Conclusions}

Most of the developed countries are currently committed with energy efficiency plans in which renewable energy sources and more efficient devices are used in order to decrease energy consumption and the associated carbon emissions. However, other technological developments such as those made possible by IoT and the Smart Cities umbrella allow further improvements. In this paper we presented a data-oriented approach for the autonomous management of public lighting networks. It is based on the acquisition of data from several sources, including luminaries, weather stations and ambient sensors. The proposed man- 
agement scheme treats each luminary individually and takes into consideration their surrounding conditions. When compared to the current management policy, the proposed approach leads to a decrease of $28 \%$ in energy consumption while still maintaining the lighting levels defined in the European norm for the corresponding zone.

\section{Acknowledgments}

This work is co-funded by Fundos Europeus Estruturais e de Investimento (FEEI) through Programa Operacional Regional Norte, in the scope of project NORTE01-0145-FEDER-023577 and by national funds through FCT - Fundação para a Ciência e Tecnologia through projects UID/CEC/00319/2019 and UIDB/04728/2020.

\section{References}

1. Road lighting standards. Standard EN 13201:2015, European Committee for Standardization (CEN). (2015)

2. Albino, V., Berardi, U., Dangelico, R.M.: Smart cities: Definitions, dimensions, performance, and initiatives. Journal of urban technology 22(1), 3-21 (2015)

3. Bakıcı, T., Almirall, E., Wareham, J.: A smart city initiative: the case of barcelona. Journal of the knowledge economy 4(2), 135-148 (2013)

4. Barrionuevo, J.M., Berrone, P., Ricart, J.E.: Smart cities, sustainable progress. IESE Insight 14(14), 50-57 (2012)

5. Boyce, P., Fotios, S., Richards, M.: Road lighting and energy saving. Lighting Research \& Technology 41(3), 245-260 (Sep 2009), http://journals.sagepub.com/ doi $/ 10.1177 / 1477153509338887$

6. Caragliu, A., Del Bo, C., Nijkamp, P.: Smart cities in europe. Journal of urban technology 18(2), 65-82 (2011)

7. Carneiro, D., Sousa, C.: The influence of external factors on the energy efficiency of public lighting. In: 2018 Proceedings of the CAPSI'18 - 18th Conference of the Portuguese Association for Information Systems. vol. 39. CAPSI (2018)

8. Chen, T.M.: Smart grids, smart cities need better networks [editor's note]. IEEE Network 24(2), 2-3 (2010)

9. Knight, C.: Field surveys of the effect of lamp spectrum on the perception of safety and comfort at night. Lighting Research \& Technology 42(3), 313-329 (2010)

10. Mumford, E.: A socio-technical approach to systems design. Requirements Engineering 5(2), 125-133 (2000)

11. Patterson, M.G.: What is energy efficiency?: Concepts, indicators and methodological issues. Energy Policy 24(5), 377 - 390 (1996), http://www.sciencedirect.com/ science/article/pii/0301421596000171

12. Pordata: Energy consumption in portugal by type, https://www.pordata. $\mathrm{pt} /$ Portugal/Consumo + de + energia + electrica + total $+\mathrm{e}+$ por + tipo + de + consumo-1124, accessed: 2019-11-20

13. Rabaza, O., Molero-Mesa, E., Aznar-Dols, F., Gómez-Lorente, D.: Experimental Study of the Levels of Street Lighting Using Aerial Imagery and Energy Efficiency Calculation. Sustainability 10(12), 4365 (Nov 2018), http://www.mdpi. com/2071-1050/10/12/4365 